\title{
Influencia de la Oclusión en el alineamiento de las vértebras cervicales con relación al plano Silla-Nasión
}

\author{
Influence of occlusion in the alignment of the cervical vertebrae to \\ the Silla-Nasión plane
}

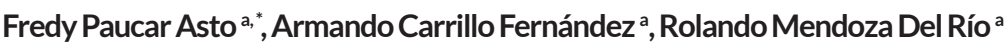 \\ a Universidad Continental, Carrera Profesional de Odontología, Huancayo, Perú
}

\section{RESUMEN}

Este trabajo tiene como objetivo general determinar los efectos que produce la oclusión dentaria en el alineamiento de las vértebras cervicales con relación al plano SN mediante el uso de una guía oclusal. Respecto a la metodología fue explicativo, transversal y pre experimental; para la obtención de datos se utilizó una guía oclusal por una semana y un radiografía cefalometrica antes y después del uso del dispositivo oclusal para medir la nueva posición de las vértebras a nivel de la apófisis odontoides de la segunda vertebra cervial tanto angular y lineal en relación al plano SN. La muestra fue de un grupo de estudio de 10 estudiantes. Entre los resultados obtenidos se puede observar que el ángulo formado por el eje de la apófisis odontoides (C2) y el plano $\mathrm{S}-\mathrm{N}$ al cual denominaremos ángulo $\mathrm{SN}-\mathrm{O}$, en oclusión habitual en promedio es de $93,35^{\circ}$ y en relación céntrica en promedio es de $101,65^{\circ}$, obteniendo una diferencia de $8,3^{\circ}$ entre ambas posiciones mandibulares. En cuanto a las mediciones lineales para la oclusión habitual una distancia promedio de 26,1 mm y de $18,9 \mathrm{~mm}$ para la posición en relación céntrica. Existiendo una diferencia de 7,2 mm para ambas posiciones mandibulares. En conclusión, existe influencia de la Oclusión Dentaria en el alineamiento de las vértebras cervicales con relación al plano SN.

Palabras clave: Relación céntrica, relación habitual, postura cervical.

\begin{abstract}
This research has studied the effect of occlusion in the cervical alignment of vertebrae with relation to the SN plane, by the use of an oclusal guide. The methodology was explicative, transversal and pre experimental. A study group of 10 university students was the sample, to obtain the data base, we used an oclusal guide for one week and radiographic capture to measure the new position of the first 2 vertebrae; angular and linearly position in relation to the point SN. The results show the angle formed by the axis of the odontoid apophysis (C2) and the plane S-N, which we named angle $\mathrm{SN}-\mathrm{OR}$; in habitual occlusion the average is $93,35^{\circ}$ and in central relation the average of $101,65^{\circ}$, obtaining a difference of $8,3^{\circ}$ between both pertaining to the jaw positions. . The linear measurements for the habitual occlusion shows an average distance of $26,1 \mathrm{~mm}$ and $18,9 \mathrm{~mm}$ for the position in central relation. Existing a difference of 7,2 $\mathrm{mm}$ for both pertaining to the jaw positions. As Conclusion: the Occlusion influence in the alignment of the cervical vertebrae with relation to the plane $\mathrm{SN}$.
\end{abstract}

Keywords: Central relation, habitual relation, cervical position.

Historial del artículo:

Recibido, 10 de diciembre de 2015; aceptado, 1 de febrero de 2016; disponible en línea, 30 de marzo de 2016

* Cirujano Dentista, docente de la Universidad Continental.

Correo: fpaucara@continental.edu.pe

Este es un artículo de acceso abierto, licencia CC BY (https://creativecommons.org/licenses/by/4.0/) 


\section{INTRODUCCIÓN}

La patología de la alteración cráneo mandibular, bordea el $93 \%$ de las patología más comunes de la cavidad oral (1). La falta de información sobre este tema, no permite al profesional dar a ciencia cierta una respuesta sobre la posible relación entre la oclusión y el posicionamiento de las vértebras cervicales, con la sintomatología dolorosa de la musculatura cervical asociada a esta patología, como medio efectivo de tratamiento en las disfunciones cráneo mandibular, basado hasta ahora en la percepción clínica de la mejora por parte del paciente que presenta esta patología.

El resultado del trabajo de investigación, permitirá establecer un protocolo o guía clínica, con un marco teórico científico sobre el tratamiento universal confiable y predecible para esta patología, que podrán brindarse a todos los pacientes que la padecen.

Díaz (2) menciona a Solow y Tallgreen que determinaron una fuerte asociación entre el biotipo dólicofacial y una angulación cráneo-cervical aumentada. En trabajos posteriores, demostraron que la postura de la cabeza está fuertemente influenciada por la resistencia respiratoria.

Limaylla R. (3), según Rocabado si la curvatura fisiológica de la columna cervical se rectifica $\circ$ se invierte, se produce como consecuencia una tensión de la fascia cervical insertada en el hueso hioides que provoca una tracción posterior de éste. Esta situación trae como consecuencia un descenso de la posición normal de la lengua y de la mandíbula.

Villalón P. (4), en su trabajo menciona a Katsaros que observó que la función de los músculos masticatorios influye en el crecimiento transversal del esqueleto cráneo-facial en las áreas donde ejercen su acción directa, así como en áreas de la arcada donde los molares están erupcionando. Postula que la dimensión y morfología de las suturas faciales, así como la aposición ósea en éstas, son afectadas negativamente frente a una disminución en la función masticatoria.

Según Fuentes R, Freesmeyer W, y Henríquez J. (5) en el artículo "Influencia de la postura corporal en la prevalencia de las disfunciones craneomandibulares" menciona: las disfunciones craneomandibulares (DCM) han sido estudiadas por largo tiempo, buscando el rol de los diferentes factores que participan en su etiología. Estas, se han relacionado con mal oclusiones, bruxismo y estrés. Clínicamente sin embargo, se puede apreciar que en los pacientes disfuncionados existen otras razones para las causas de sus síntomas y molestias que no provienen del sistema craneomandibular. La estabilidad ortostática del cráneo sobre la columna cervical influye en la etiología de las DCM y del dolor orofacial, porque determina la posición espacial de la mandíbula, influenciando aspectos de la oclusión, como la posición de contacto retruida, el espacio interoclusal, la posición de eje de bisagra terminal de la articulación temporomandibular (ATM) y la relación de contacto de los dientes en la oclusión habitual, además de la actividad electromiográfica de los músculos masticadores y de la nuca, que al alterarse pueden producir disfunciones del sistema craneomandibular.

Fuentes (5) cita a Thompson quien describió la influencia de la postura del cuerpo en la posición de la mandíbula.

Collado (6) menciona a Gelb quien entrega un planteamiento importante en el diagnóstico y tratamiento de las disfunciones craneomandibulares, señalando que las alteraciones de la postura juegan un rol etiológico en las DCM, y propone que el tratamiento disfuncional incluya la corrección de la postura corporal.

Fuentes (5) cita a Hansson quien plantea que una alteración de la posición de las caderas puede ser una causa etiológica para las DCM.

Garcia de Paula, Mussolino y Díaz (7) concluyen que las alteraciones de la articulación temporomandibular (ATM) son más frecuentes cuando en el mismo lado del cuerpo se presenta la cadera más baja.

Limaylla (3) cita a Bergbreiter quien encontró una relación entre la alteración de la postura de las caderas (medidas con el acromiopelvímetro de Cross) y alteraciones de la ATM y además observó que en la ATM que se encuentra en el lado del cuerpo donde existe una cadera más baja, presenta al examen clínico una mayor prevalencia de ruido articular.

Fuentes (5) afirma que los músculos masticatorios (masetero y temporal), que se encuentran en el lado del cuerpo donde existe una cadera más baja, son más sensibles a la palpación.

Chávez (8) cita a Shup \& Zernial que en su trabajo determinaron la relación entre las alteraciones posturales de caderas y hombros con la prevalencia de los signos y síntomas de las disfunciones craneomandibulares.

Restrepo, Quintero, Tamayo, Tamayo (9), mencionan que en la función del sistema cráneomandibular se reconoce como un factor importante la postura de la cabeza sobre la columna cervical. La estabilidad ortostática del cráneo sobre la columna cervical permite un correcto funcionamiento de este sistema.

Es en este contexto es que surge el interés por explicar 
si existe influencia de la oclusión en el alineamiento de las vértebras cervicales con relación al plano SN (silla-nasión) .

El problema general fue ¿̇ué efectos produce la oclusión en el alineamiento de las vértebras cervicales con relación al plano SN?. Siendo el objetivo general; explicar que efectos produce la oclusión en el alineamiento de las vértebras cervicales con relación al plano SN.
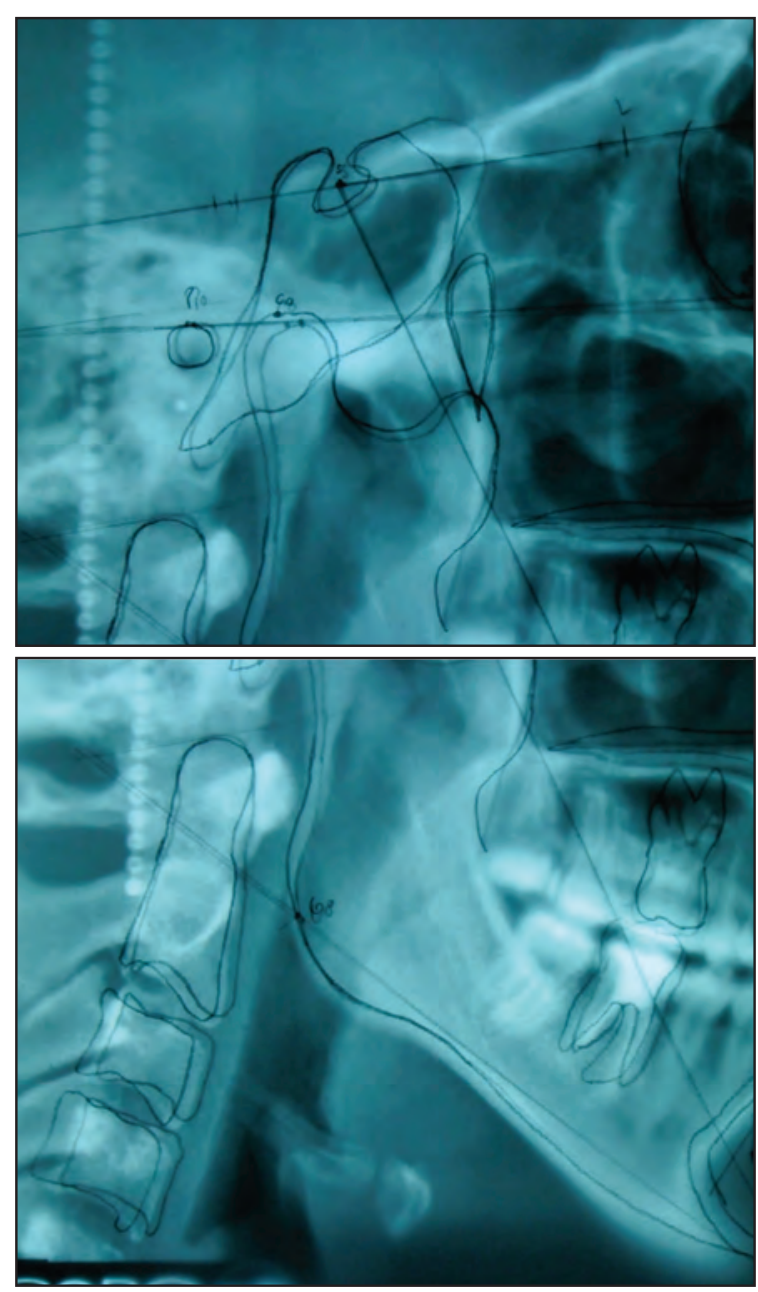

Figura $N^{\circ} 1$ : Radiografías superpuestas del mismo paciente, se observa cómo cambia la posición del maxilar inferior a nivel condilar de oclusión habitual a céntrica y el cambio de las vértebras cervicales.

La investigación toma importancia debido a que no existe un protocolo que explique y de pautas de como la oclusión puede estar relacionada con la posición de las vértebras cervicales, ante la presencia de la sintomatología asociados a trastornos cráneo mandibulares como, dolor del cuello y el dolor de brazos y región pectoral por compresión del plexo nervioso Braquial, que pasa en el cuello entre los músculos escalenos anterior y medio y que estos tienen inserción en las vértebras cervicales y al cambiar la posición de la oclusión, estas cambiara la longitud fisiológica de los músculos de la región cervical, cambiado así su fisiología y disminuyendo o desapareciendo la sintomatología.

\section{MATERIAL Y MÉTODOS}

Los pacientes evaluados fueron finalmente 10, los datos fueron recogidos con la técnica modificada de Peter Dawson para ver si tenían uno o dos arcos de cierre, luego del cual se examinó, los movimientos excursivos mandibulares, se anotó las interferencias oclusales tanto en céntrica como en excéntrica, palpación muscular y articular que incluyo la palpación de los músculos pterigoideos externos fascículos inferiores.

Se utilizó, radiografías cefalométrica de perfil estricto, en oclusión habitual, y luego una segunda placa después de utilizar por al menos una semana un deprogramador anterior, Jick de Lucia, sobre las placas obtenidas, se realizó un calco cefalométrica dirigido hacia la base craneal anterior, agujero auditivo externo, y las vértebras de la región cervical observables en las radiografías, primero en la placa en oclusión habitual, para después superponer el calco en la segunda placa y hacer coincidir las estructuras anatómicas de la base craneal anterior, para luego calcar la nueva posición de la columna cervical, obtenida esta información se procedió a marcar el eje mayor de la apófisis odontontoides de la segunda vértebra cervical, realizando dos mediciones tanto en la parte superior e inferior de la apófisis en el plano horizontal obteniendo la media de estas medidas, procediendo a unir estos puntos y proyectarlos sobre el plano SN y realizar la medición de los ángulos que forman la proyección de la apófisis odontoides en las posiciones de oclusión habitual y en relación céntrica, también se realizó una medición lineal de la proyección en el plano S-N desde el punto $\mathrm{S}$.

El diseño de la investigación fue pre experimental, con un solo grupo con pre y pos test.

Esquema: GE: O1 -.-- X --.- O2

\section{RESULTADOS}

En la tabla $\mathrm{N}^{\circ} 01$ podemos visualizar la variación en grados desde la primera medida a la segunda medida tomada a los 07 dias, todas en tendencia al aumento.

En la tabla $N^{\circ} 02$ se muestra la variación en mm desde la primera medida a la segunda medida tomada a laos 07 días, todas en tendencia a la disminución. 
Tabla $N^{\circ}$ 1: Medida del ángulo SN-Apófisis Odontoides de la segunda vértebra cervical.

\begin{tabular}{ccc}
\hline Paciente & Primera medida & $\begin{array}{c}\text { Segunda medición 07 } \\
\text { días después }\end{array}$ \\
\hline 1 & $100,00^{\circ}$ & $108,00^{\circ}$ \\
2 & $100,00^{\circ}$ & $106,00^{\circ}$ \\
3 & $98,00^{\circ}$ & $102,50^{\circ}$ \\
4 & $79,00^{\circ}$ & $86,00^{\circ}$ \\
5 & $84,00^{\circ}$ & $97,00^{\circ}$ \\
6 & $92,00^{\circ}$ & $101,00^{\circ}$ \\
7 & $89,00^{\circ}$ & $102,00^{\circ}$ \\
8 & $96,00^{\circ}$ & $105,00^{\circ}$ \\
9 & $90,50^{\circ}$ & $98,00^{\circ}$ \\
10 & $105,00^{\circ}$ & $111,00^{\circ}$ \\
\hline
\end{tabular}

Tabla $N^{\circ}$ 2: Distancia lineal desde el punto SN a la Apófisis Odontoides de la segunda vértebra cervical.

\begin{tabular}{ccc}
\hline Paciente & Primera medida & $\begin{array}{c}\text { Segunda medición 07 } \\
\text { días después }\end{array}$ \\
\hline 1 & $18,00 \mathrm{~mm}$ & $12,00 \mathrm{~mm}$ \\
2 & $18,00 \mathrm{~mm}$ & $12,00 \mathrm{~mm}$ \\
3 & $25,00 \mathrm{~mm}$ & $20,50 \mathrm{~mm}$ \\
4 & $33,00 \mathrm{~mm}$ & $26,00 \mathrm{~mm}$ \\
5 & $33,50 \mathrm{~mm}$ & $20,00 \mathrm{~mm}$ \\
6 & $24,00 \mathrm{~mm}$ & $17,00 \mathrm{~mm}$ \\
7 & $34,00 \mathrm{~mm}$ & $25,00 \mathrm{~mm}$ \\
8 & $24,00 \mathrm{~mm}$ & $18,50 \mathrm{~mm}$ \\
9 & $28,00 \mathrm{~mm}$ & $22,50 \mathrm{~mm}$ \\
10 & $23,50 \mathrm{~mm}$ & $15,50 \mathrm{~mm}$ \\
\hline
\end{tabular}

Tabla $N^{\circ}$ 3: Distancia lineal desde el punto SN a la Apófisis Odontoides de la segunda vértebra cervical.

\begin{tabular}{lccc}
\hline & N & Correlation & Sig. \\
\hline $\begin{array}{l}\text { Segunda después de 07 dias } \\
\text { medida en ángulos \& Primera }\end{array}$ & 10 & 0,937 & 0,000 \\
medida en ángulos & & & \\
\hline
\end{tabular}

En la tabla $N^{\circ} 03$ se visualiza el grado de correlación entre los datos de las medidas en ángulos.

La tabla $N^{\circ} 04$ muestra el resultado de la Prueba T de Student para variables relacionadas consideración medida en ángulos.

La tabla $N^{\circ} 05$ muestra el grado de correlación entre los datos de las medidas en $\mathrm{mm}$.

La tabla $\mathrm{N}^{\circ} 06$ muestra el resultado de Sig. (2-tailed) 0,000 donde se acepta la hipótesis que existe influencia de la Oclusión en el alineamiento de las vértebras cervicales con relación al plano SN de la medición en milímetros.

\section{DISCUSIÓN}

La investigación reafirma los datos del MINSA (1) que la alteración cráneo mandibular, bordea el $93 \%$ de las patología más comunes de la cavidad oral.

Se evidenció con mayor precisión los datos de Ramón (5) que menciona: la estabilidad ortostática del cráneo sobre la columna cervical influye en la etiología de las DCM y del dolor orofacial, porque determina la posición espacial de la mandíbula, influenciando aspectos de la oclusión dentaria, como la posición retruida de contacto, el espacio interoclusal, la posición de eje de bisagra terminal de la articulación temporomandibular (ATM) y la relación de contacto

Tabla $N^{\circ}$ 5: Distancia lineal desde el punto SN a la Apófisis Odontoides de la segunda vértebra cervical.

\begin{tabular}{lccc}
\hline & N & Correlation & Sig. \\
\hline $\begin{array}{l}\text { Primera medida en } \\
\text { milímetros \& Segunda }\end{array}$ & 10 & 0,904 & 0,000 \\
medida en milímetros & & & \\
\hline
\end{tabular}

Tabla № 4: Distancia lineal desde el punto SN a la Apófisis Odontoides de la segunda vértebra cervical.

\begin{tabular}{|c|c|c|c|c|c|c|c|c|}
\hline & \multicolumn{5}{|c|}{ Paired Differences } & \multirow{3}{*}{$t$} & \multirow{3}{*}{$d f$} & \multirow{3}{*}{$\begin{array}{c}\text { Sig. } \\
\text { (2-tailed) }\end{array}$} \\
\hline & \multirow{2}{*}{ Mean } & \multirow{2}{*}{$\begin{array}{l}\text { Std. } \\
\text { Devia- } \\
\text { tion }\end{array}$} & \multirow{2}{*}{$\begin{array}{l}\text { Std. } \\
\text { Error } \\
\text { Mean }\end{array}$} & \multicolumn{2}{|c|}{$\begin{array}{c}95 \% \text { Confidence } \\
\text { Interval of the Difference }\end{array}$} & & & \\
\hline & & & & Lower & Upper & & & \\
\hline $\begin{array}{l}\text { Segunda } \\
\text { después de } 07 \\
\text { dias medida } \\
\text { en ángulos - } \\
\text { Primera medida } \\
\text { en ángulos }\end{array}$ & 8,3000 & 2,84019 & 0,89815 & 6,26825 & 10,33175 & 9,241 & 9 & 0,000 \\
\hline
\end{tabular}


Tabla $N^{\circ}$ 6: Distancia lineal desde el punto SN a la Apófisis Odontoides de la seguna vértebra cervical.

\begin{tabular}{|c|c|c|c|c|c|c|c|c|}
\hline & \multicolumn{5}{|c|}{ Paired Differences } & \multirow{3}{*}{$\dagger$} & \multirow{3}{*}{ df } & \multirow{3}{*}{$\begin{array}{l}\text { Sig. } \\
\text { (2-tailed) }\end{array}$} \\
\hline & \multirow{2}{*}{ Mean } & \multirow{2}{*}{$\begin{array}{l}\text { Std. } \\
\text { Devia- } \\
\text { tion }\end{array}$} & \multirow{2}{*}{$\begin{array}{l}\text { Std. } \\
\text { Error } \\
\text { Mean }\end{array}$} & \multicolumn{2}{|c|}{$\begin{array}{c}95 \% \text { Confidence } \\
\text { Interval of the Difference }\end{array}$} & & & \\
\hline & & & & Lower & Upper & & & \\
\hline \multirow{2}{*}{$\begin{array}{l}\text { Primera medida } \\
\text { en milímetros } \\
\text { - Segunda } \\
\text { medida en } \\
\text { milímetros }\end{array}$} & 7,20 & 2,57337 & 0,81377 & 5,35912 & 9,04088 & & 9 & 0,000 \\
\hline & 0,00 & & & & & 8,848 & & \\
\hline
\end{tabular}

de los dientes en la oclusión habitual, además de la actividad electromiográfica de los músculos masticadores y de la nuca, que al alterarse pueden producir disfunciones del sistema craneomandibular.

En la investigación se observó clínicamente lo que menciona Garcia de Paula, Mussolino y Díaz (7); que las alteraciones de la articulación temporomandibular (ATM) son más frecuentes cuando en el mismo lado del cuerpo se presenta la cadera más baja.

Contrariamente a Fuentes (5) Collado (6), los resultados clínicos obtenidos en la investigación indican que la posición de la mandíbula modifica parte de la posición de la columna.

Los resultados de la investigación reafirman al trabajo de Restrepo, Quintero, Tamayo, Tamayo (9) que resume: la función del sistema cráneomandibular se reconoce como un factor importante la postura de la cabeza sobre la columna cervical. La estabilidad ortostática del cráneo sobre la columna cervical permite un correcto funcionamiento de este sistema.

Las conclusiones son:

Existe influencia de la oclusión dentaria en el alineamiento de las vértebras cervicales con relación al plano SN, medida en la proyección de la apófisis Odontoides y el plano S-N.

A partir de los resultados ángulo $\mathrm{SN}-\mathrm{O}(\mathrm{h})$ de $93,5^{\circ}$ y un ángulo $\mathrm{SN}-\mathrm{O}$ (c) de $101.65^{\circ}$, podemos utilizarlos como parámetro objetivo tanto para el diagnóstico así como para controlar la evolución del tratamiento de la Disfunción Cráneo Mandibular. La investigación toma importancia, ya que no existe un protocolo que explique y de pautas de como la oclusión dentaria puede estar relacionada con la posición de las vértebras cervicales, ante la presencia de la sintomatología asociados a trastornos cráneo mandibulares como, dolor del cuello, hombros y el dolor de brazos y región pectoral por compresión del plexo nervioso Braquial, lo que provocaría la compresión de este plexo nervioso que simularía un síndrome compresivo por disminución de los espacios intervertrebrales, lo que no observaría en una radiografía haciendo muy confuso su diagnóstico, por lo tanto si nosotros llevamos la mandíbula a una posición fisiológica (Relación Céntrica) las vértebras retomaran su posición fisiológica por ende los músculos, desparecerá la compresión del plexo braquial y desaparecerán los síntomas en el paciente.

El resultado del trabajo de investigación, permitirá establecer un protocolo o guía clínica, con un marco teórico científico sobre el tratamiento universal confiable y predecible para esta patología, que podrán brindarse a todos los pacientes que la padecen. Esta medida $\mathrm{SN}-\mathrm{O}$ se puede realizar en los estudios cefalométricos de los pacientes con necesidad de ortodoncia y de esta manera evaluar la relación céntrica sin manipular al paciente.

El presente trabajo de investigación contribuye a esclarecer la etipoatogenia de los síntomas asociados a la disfunción craneomandibular de una manera objetiva a través de radiografías cefgalometricas

\section{REFERENCIAS BIBLIOGRÁFICAS}

1. Ministerio de Salud [Internet]. Lima: MINSA; 2014 [citado el 10 de octubre de 2015]. Prevención para la salud [aprox. 2 pantallas]. Disponible en: http://www.minsa.gob.pe/ index.asp? op $=2$

2. Díaz K. Análisis biomecánico craneocervical a través de una telerradiografía lateral. Rev. Chil. Ortod. 1984; 1: 42-52.

3. Limaylla R. Transtornos temporomandibulares y alteraciones postulares de la columna cervical en personal asistencial del departamento de Odontología del Hospital Militar Central. [Tesis de pregrado]. Lima: Universidad Nacional Mayor de San Marcos; 2007.

4. Villalón P, Frugone R, Palomino H. Algunas Mediciones Radiográficas Cráneo - Cervicales según Biotipo de Ricketts. Revista dental de Chile. 2004; 95(3): $11-17$.

5. Fuentes R, Freesmeyer W, Henríquez J. Influencia de la postura corporal en la prevalencia de las disfunciones craneomandibulares. Rev. méd. Chile. 1999; 127(9): 1079-1085. 
6. Collado S. Estudio morfométrico de la articulación temporomandibular en radiología. Rev fisioter. 2007; 6(1): 5-12.

7. Garcia de Paula F, Mussolino A y Díaz K. Alteraciones posturales y su repercusión en el sistema estomatognática. Acta odontol. Venez. 2008; 26(4): 517-522.

8. Chávez, E. Relación entre alteraciones posturales y maloclusiones observadas en pacientes entre 10 a 16 años de edad que asistieron a la clínica de odontología de la Universidad Mayor, Real y Pontificia de San Francisco Xavier de Chuquisaca [Tesis de posgrado]. Sucre: Universidad Mayor, Real y Pontificia San Francisco Xavier de Chuquisaca; 2010.

9. Restrepo C, Quintero Y, Tamayo M, Tamayo V. Efecto de la posición craneocervical en las funciones orales fisiológicas. Revista CES Odontología. 2008; 21 (1): 71-75. 